\title{
Seroprevalencia de Leptospirosis en niños de zonas inundables cíclicamente de Asunción, Paraguay
}

\section{Leptospirosis seroprevalence in children living in cyclically flooded areas in Asuncion, Paraguay}

\author{
Angélica Leonor Correa Fretes ${ }^{(1)}$, Cinthia Lugo Jara ${ }^{(1)}$, Ludmila Aramí Lezcano Rodas ${ }^{(1)}$, Gustavo \\ Ariel Meaurio Bogado ${ }^{(1)}$, Gloria Celeste Samudio Domínguez ${ }^{(2)}$, Andrés Canese ${ }^{(3)}$
}

\section{RESUMEN}

Introducción: La leptospirosis es una enfermedad infectocontagiosa, causada por Leptospira. La enfermedad puede variar de leve a severa, pudiendo incluso ocasionar la muerte. Material y Métodos: Estudio observacional, descriptivo de corte transverso de seroprevalencia, realizado en setiembre de 2014 . Se incluyeron niños sanos de 5 a 15 años de edad habitantes de zonas inundables. A los mismos se le aplicó un cuestionario, y luego de consentimiento informado de los padres, se procedió a la extracción de sangre para realizar prueba diagnóstica. Resultados: Se incluyeron en el estudio 80 niños a los que se les realizó búsqueda de anticuerpos anti-Leptospira, del tipo IgG por el método de ELISA y a 62 niños se aplicó la encuesta de factores de riesgo y enfermedad sintomática probable. Todos ellos entraron en contacto con agua en las inundaciones al menos una vez, todos refirieron contactos con animales, visualizando ratas alrededor de los domicilios. De los 80 niños a los que se practicó ELISA, sólo 3 fueron positivos (3,6\%). Conclusiones: La seroprevalencia de leptospirosis en población pediátrica de zonas inundables es baja en zona ribereña de Asunción, sin embargo debe ser implementada una vigilancia activa a fin de detectar los casos en forma temprana.

Palabras clave: Leptospirosis, seroprevalencia, zona indudable, pediatría.

\begin{abstract}
Introduction: Leptospirosis is an infectious disease caused by Leptospira. The disease can range from mild to severe, and can even cause death. Materials and Methods: We performed an observational, descriptive, crosssectional study of seroprevalence, made in healthy children, ages 5 to 15 years, living in flood prone areas during September 2014. The study subjects answered a questionnaire and, after obtaining informed consent from the parents, we proceeded to extract blood for diagnostic testing. Results: We studied 80 children for antiLeptospira IgG antibodies using ELISA and 62 children's assessed their risk factors and symptomatology for probable clinical disease. All study subjects reported coming into contact with flood waters at least once, and all reported contacts with animals, including seeing rats around their homes. Of the 80 children who underwent ELISA, only 3 were positive (3.6\%). Conclusions: Seroprevalence of leptospirosis in this pediatric population living in flood prone areas of Asunción is low, but active surveillance must be implemented for early detection of cases.
\end{abstract}

Keywords: Leptospirosis, seroprevalence, flood-prone area, pediatrics.

\footnotetext{
1. Residente de Pediatría del Hospital General de Barrio Obrero, Ministerio de Salud Pública y Bienestar Social. Asunción, Paraguay.

2. Infectóloga Pediatra del Hospital General de Barrio Obrero, Ministerio de Salud Pública y Bienestar Social. Asunción, Paraguay.

3. Microbiólogo, Cátedra de Microbiología de la Facultad de Ciencias Médicas, Universidad Nacional de Asunción. San Lorenzo, Paraguay.

Correspondencia: Gloria Celeste Samudio Domínguez.E-mail: gsamudio.samudio@gmail.com

Recibido:28/04/2015; Aceptado: 16/10/2015.

Los autores declaran que no existe ningún conflicto de interés. El costo del presente estudio fue cubierto en su totalidad por los investigadores. http://dx.doi.org/10.18004/ped.2015.diciembre.211-215
} 


\section{INTRODUCCIÓN}

La leptospirosis es una enfermedad infectocontagiosa, causada por microorganismos del género Leptospira. Inicialmente fueron reconocidas dos especies, la forma patógena Leptospira interrogans y la saprofita Leptospira biflexa. Se considera entre las enfermedades remergentes. Puede presentarse en países industrializados o en vías de desarrollo ${ }^{(1)}$.

Recientemente, varias especies de Leptospira han sido diferenciadas sobre la base de la relación entre sus ADN. Se han descrito alrededor de 200 serovares patógenos, los que han sido agrupados en 25 serogrupos en base a sus similitudes antigénicas ${ }^{(2)}$.

La enfermedad puede variar de leve a severa, pudiendo incluso ocasionar la muerte. La infección en humanos ocurre a través del contacto con piel o mucosas, con agua o suelo húmedo contaminado con orina infectada de animales ${ }^{(3-6)}$.

Las lesiones de piel, con pérdida de sustancia, facilitan la infección pero no existen estudios que cuantifiquen la relación entre ambos ${ }^{(7,8)}$. Inundaciones y lluvias copiosas, no usar calzados, lavar la ropa o utensilios en agua de ríos o arroyos, algunas ocupaciones como granjería, trabajar limpiando alcantarillas, minería, trabajar con animales o en actividades militares son todas actividades y ocupaciones de riesgo para contraer la enfermedad ${ }^{(7,9-12)}$.

Los reservorios son principalmente ratas, perros, cerdos, ganado vacuno. Las personas que viven en zonas inundables y en malas condiciones socioeconómicas son candidatas a padecer la enfermedad ${ }^{(9-15)}$.

La forma sintomática de la infección por Leptospira se caracteriza por fiebre acompañada de otros síntomas tales como cefalea, sufusión conjuntival, dolores musculares y meningismo. Algunas personas pueden tener infección inaparente o síntomas muy leves, por lo que muchas veces la enfermedad puede pasar desapercibida, sobre todo en áreas donde la enfermedad es endémica ${ }^{(16)}$.

La ciudad de Asunción se caracteriza por poseer habitantes que se encuentran en cinturones de pobreza en la ribera del río, por lo que sufren de inundaciones periódicas. Además de vivir en condiciones precarias de higiene, tienen contacto con perros y también con ratas (Figura 1).

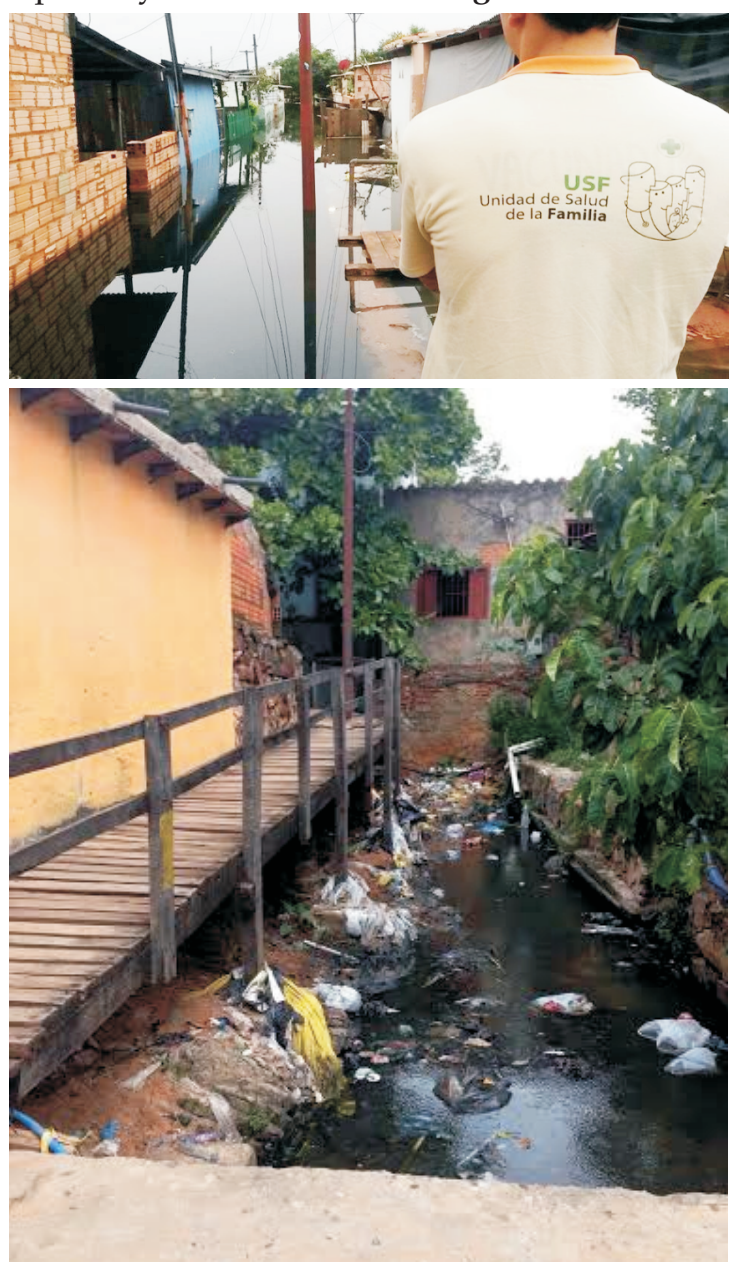

Figura 1. Zonas ribereñas en donde se encuestó y tomó material a los niños que integraron el estudio.

Estas condiciones favorecen el ambiente para la aparición de leptospirosis. No se conoce la prevalencia de la enfermedad en nuestro país, por lo que planteamos un estudio de seroprevalencia de esta enfermedad en población pediátrica.

\section{MATERIALES Y MÉTODOS}

Se realizó un estudio observacional, descriptivo, prospectivo de corte transverso. Previo consentimiento informado, ingresaron al estudio niños de 5 a 15 años de edad, que habitan en zonas inundables de la ciudad de Asunción. Para ello, médicos pediatras en formación realizaron trabajo de campo, el cual consistió en visitas domiciliarias a las familias que viven en zonas inundables. A las mismas se aplicó cuestionario con variables de edad, sexo, número de inundaciones que sufrieron, 
contacto con animales, número de internaciones hospitalarias que tuvieron y causas de las mismas. Además se realizó extracción de muestra sanguínea para realización de la serología, para búsqueda cualitativa de anticuerpos anti-Leptospira del tipo IgG, por la metodología de ELISA (Enzyme Linked Immunosorbent Assay, análisis de inmunoabsorción ligado a enzimas), método validado frente a la prueba de referencia de microaglutinación (MAT, por sus siglas en inglés) con $5 \times 10^{7}$ leptospiras $/ \mathrm{ml}$, con una sensibilidad no valorada por falta de correlación entre la metodología MAT y ELISA y una especificidad del $97 \%$.

\section{RESULTADOS}

El estudio tuvo dos componentes: encuesta para conducta de riesgo y extracción de sangre para estudio serológico. En el primer componente participaron 62 padres de niños y en el segundo componente (extracción de material para estudio de serología) participaron 80 niños.

Para el primer componente, sólo 62 de ellos aceptaron participar de la encuesta, pero se permitió la extracción de sangre de un total de 80 pacientes pediátricos con la finalidad de realizar la búsqueda de anticuerpos anti-Leptospira por ELISA.

La muestra de 62 pacientes estuvo conformada por $35(56 \%)$ niñas y $27(44 \%)$ varones. El promedio de edad fue de 10,3 años, mediana de 11 años, moda de 14. Con mínima de 5 y máxima de 15 años de edad.

Todos los niños habían sufrido al menos una inundación en sus vidas, hasta un máximo de 3 inundaciones. En todos los casos los padres refirieron contacto de los niños (piel expuesta) con el agua proveniente de la inundación, en forma repetida, aunque no se midió si la misma fue en forma sostenida. Esto puede observarse en la figura 2.

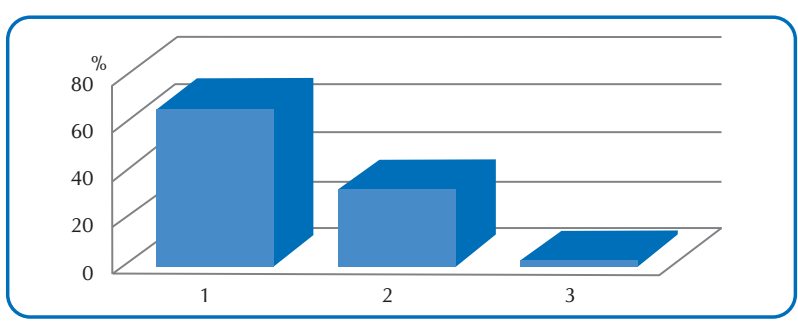

Figura 2. Distribución de niños investigados para seroprevalencia de leptospirosis de zona ribereña, según número de inundaciones. $\mathrm{N}=62$.
También todos ellos tienen contacto y/o poseen animales, específicamente perros y gatos como animales domésticos. Además, todos refirieron al menos visualizar ratas en las cercanías o dentro de la vivienda.

A 80 niños se les realizó la búsqueda de anticuerpos del tipo IgG anti-Leptospira, obteniéndose un resultado positivo en 3 personas, lo cual significa una seroprevalencia del 3,6\%. Estos casos fueron al parecer de expresión clínica asintomática a leve, ya que los padres de 2 niños no refirieron internación por ningún tipo de afectación, solamente 1 de ellos refirió sintomatología compatible con una leptospirosis leve.

\section{DISCUSIÓN}

La leptospirosis es una enfermedad potencialmente letal. Su endemicidad está favorecida por la elevada densidad poblacional y condiciones sanitarias deficientes o inexistentes. En Paraguay no se había realizado, hasta la fecha, ningún estudio en áreas que pueden ser consideradas de riesgo, tales como las zonas ribereñas, inundables en forma cíclica, donde además de necesidades básicas insatisfechas, se conjugan la convivencia con animales portadores, tales como ratas y canes. La seroprevalencia varía mucho de un país a otro, de $6 \%$ hasta $69 \%{ }^{(17-20)}$.

Todas las prevalencias de la región se encuentran muy por encima de la reportada en este trabajo. Esto podría deberse a diversos factores, entre los cuales habría que tener en cuenta el pequeño tamaño muestral, que se haya realizado sólo en pacientes pediátricos lo cual deja fuera a una franja importante de adultos que podrían ser seropositivos, ya que tienen la oportunidad de haber estado más expuestos a los gérmenes o bien, y tal vez lo más alentador, es que esta espiroqueta aún no se ha instalado en el país. Esto explicaría la casi ausencia de casos de internación, aún entre los pacientes seropositivos $^{(21)}$.

El consumo agua de río se asocia a la infección por Leptospira. Habitualmente las personas en estas áreas no hierven el agua para consumirla y también la usan para el aseo personal y el lavado de la ropa ${ }^{(22)}$. Otra forma de ingreso de la bacteria puede ser a través de la mucosa nasal, oral u ocular ${ }^{(23)}$.

Desafortunadamente, en este estudio, no pudimos 
determinar la forma de ingreso de la bacteria en el organismo de los seropositivos.

Además de las vías ya citadas, el microrganismo también puede entrar por la piel, con algún tipo de lesión o abrasión, relación con la actividad de la población, ocupacional o recreacional, haciendo que la vía de ingreso para Leptospira sea más fácil ${ }^{(9,22,23)}$. Este factor estuvo presente en toda la población estudiada, sin embargo a pesar de que todos ellos tuvieron contacto con agua proveniente del rio por las inundaciones en al menos una ocasión en sus cortas vidas, la prevalencia no es elevada ${ }^{(24,25)}$.

Otro factor importante es el contacto con canes, ya que los mismos son reservorios de la bacteria ${ }^{(23-25)}$. En nuestra población todos los participantes admitieron poseer o al menos estar en contacto permanente con cánidos, y visualizar frecuentemente ratas en las inmediaciones o interior de las viviendas. Sin embargo, no hemos tomado muestras de los animales, dato que sería de gran valor para determinar la fuente de Leptospira y poder actuar sobre este factor predisponente.

En nuestro país existe un solo reporte de leptospirosis sintomática, con un cuadro leve ${ }^{(26)}$. En nuestro conocimiento, este es el primer reporte de seroprevalencia de este germen en población pediátrica en el país.

\section{REFERENCIAS}

1. Bharti AR, Nally JE, Ricaldi JN, Matthias MA, Diaz MM, Lovett MA, Levett PN, Gilman RH, Willig MR, Gotuzzo E, Vinetz JM. Leptospirosis: a zoonotic disease of global importance. Lancet Inf Dis. 2003;3(12):757-71.

2. Gómez Mateos JM, Pumarola Suñé T. Leptospirosis. En: Ausina Ruiz V, Moreno Guillén S. Tratado SEIMC de enfermedades infecciosas y microbiología clínica. España: Editorial Médica Panamericana; 2006. p. 541-45.

3. Benenson A. Leptospirosis. In: Benenson A, (editor). Control of communicable diseases annual. 16 ed. Baltimore: United Book Press; 1995. p. 267-70.

4. Farr RW. Leptospirosis. Clin Infect Dis. 1995;21:1-6.

5. Faine S. Leptospirosis. In: Hausler WJ, Sussman M,
Debemos mencionar que las debilidades de este estudio es el número pequeño del tamaño muestral que podría haber incidido en la prevalencia encontrada, además no se realizó el estudio de los reservorios, dato de suma importancia para realizar predicciones de brotes futuros en humanos.

\section{CONCLUSIONES}

La seroprevalencia de leptospirosis en niños de zonas ribereñas es baja $(3,6 \%)$, con un solo cuadro sintomático, aparentemente leve. A pesar de contar con todas las condiciones favorables para padecer de esta patología, la misma se presenta con baja prevalencia.

Es una patología que debe ser vigilada, ya que los cuadros graves pueden aparecer en cualquier momento. Es necesario plantear estudios de prevalencia en animales que pueden actuar como reservorios.

\section{AGRADECIMIENTOS}

Al Dr. Julio Rolón, Director General del Hospital barrio Obrero, Dr Santiago Insaurralde, Jefe del laboratorio del Hospital Barrio Obrero, Lic. Justa Mosquiera, Lic. Blanca Ocampos, Lic. María Antonia González, de la Unidad de Salud Familiar "3 de Febrero" por su gran apoyo en la realización del presente estudio.

(editors). Topley and Wilson's microbiology and microbial infections. 9 ed. London: Arnold Press; 1998. p. 849-69.

6. Tappero J, Ashford D, Perkins B. Leptospirosis. In: Mandell G, Bennet J, Dolin R, (editors). Principles and practice of infectious diseases. 5 ed. New York: Churchill Livingstone; 1999. p. 2495-501.

7. Chan HL. Bacterial infections of the skin II: cutaneous clues to systemic infections. Ann Acad Med Singapore. 1983;12:98-102.

8. Sasaki DM, Pang L, Minette HP, Wakida CK, Fujimoto WJ, Manea SJ, Kunioka R, Middleton CR. Active surveillance and risk factors for leptospirosis in Hawaii. Am J Trop Med Hyg. 1993;48(1):35-43. 
9. Corwin A, Ryan A, Bloys W, Thomas R, Deniega B, Watts D. A water-borne outbreak of leptospirosis among United States military personnel in Okinawa, Japan. Int J Epidemiol. 1990;19:743-48.

10. Everard CO, Bennett S, Edwards CN, Nicholson GD, Hassell TA, Carrington DG, Everard JD. An investigation of some risk factors for severe leptospirosis on Barbados. J Trop Med Hyg. 1992;95(1):13-22.

11. Kupek E, de Sousa Santos Faversani MC, de Souza Philippi JM. The relationship between rainfall and human leptospirosis in Florianópolis, Brazil, 1991-1996. Braz J Infect Dis. 2000;4:131-34.

12. Plank R, Dean D. Overview of the epidemiology, microbiology, and pathogenesis of Leptospira spp in humans. Microbes Infect. 2000;2:1265-76.

13. Centers for Disease Control and Prevention. Outbreak of acute febrile illness among participants in Eco Challenge Sabah 2000-Malaysia, 2000. JAMA. 2000;284:1646.

14. Terry J, Trent M, Bartlett M. A cluster of leptospirosis among abattoir workers. Commun Dis Intell. 2000;24:15860 .

15. Bovet P, Yersin C, Merien F, Davis CE, Perolat P. Factors associated with clinical leptospirosis: a population-based case-control study in the Seychelles (Indian Ocean). Int J Epidemiol. 1999;28:583-90.

16. Ashford D, Kaiser R, Spiegel R, Perkins BA, Weyant RS, Bragg SL, Plikaytis B, Jarquin C, De Lose Reyes JO, Amador JJ. Asymptomatic infection and risk factors for leptospirosis in Nicaragua. Am J Trop Med Hyg. 2000;63(5-6):249-54.

17. Romero MH, Sánchez JA, Hayek LC. Prevalencia de anticuerpos contra Leptospira en población urbana humana y canina del Departamento del Tolima. Rev Salud Pública. 2010;12(2):268-75.
18. Céspedes M, Ormaeche M, Condori P, Balda L, Glenny M. Prevalencia de Leptospirosis y factores de riesgo en personas con antecedente de fiebre en la provincia de Manu, Madre de Dios, Perú. Rev Peru Med Exp Salud Publica. 2003;20(4):180-85.

19. García Masaya ML, Herrera García ME, Pérez Vásquez AM, Castillo Signor LC, Kestler Ordóñez RO. Seroprevalencia de leptospirosis humana en un asentamiento del área urbana de la ciudad de Guatemala. Rev Cubana Med Trop. 2013;65(2): 166-76.

20. Rodríguez-B H, Lozano-M C, Bedoya-S C, Grondona-P L. Prevalencia de leptospirosis en humanos en la zona urbana del municipio de Puerto Libertador, Córdoba, Colombia. RIAA. 2009;0(1): 23-28.

21. Organización Panamericana de la Salud. El control de las enfermedades transmisibles en el hombre. 18 ed. Uruguay: Ministerio de Salud Pública; 2005. p.807.

22. Haake D, Dundoo M, Cader R, Kubak B, Hartskeerl R, Sejvar J, Ashford DA. Leptospirosis, water sports, and chemoprophylaxis. Clin Infect Dis. 2002;34(9):e40-43.

23. Tangkanakul W, Tharmaphornpil P, Plikaytis B, Bragg S, Poonsuksombat D, Choomkasien P, Kingnate D, Ashford DA. Risk factors associated with leptospirosis in northeastern Thailand, 1998. Am J Trop Med Hyg. 2000;63(3-4):204-208.

24. Liceras J, Valdivia S. Higuchi E. Leptospirosis en el Perú. En: Anales Seminario Nacional Zoonosis y Enfermedad de Transmisión Alimentaria. Lima: Ministerio de Salud; 1989. p.7-20.

25. Acha P, Szyfres B. 2001. Zoonosis y enfermedades transmisibles al hombre y a los animales. 3 ed. Washington: Organización Panamericana de la Salud; 2001.

26. Samudio-D GC, Cuevas C, Brizuela-E S, Coronel J. Leptospirosis en pediatría: a propósito de un caso. Pediatr (Asunción). 2010;37(1):48-51. 